\title{
The Criticality of Spare Parts Evaluating Model Using Artificial Neural Network Approach
}

\author{
Lin Wang ${ }^{1}$, Yurong Zeng ${ }^{2}$, Jinlong Zhang ${ }^{1}$, Wei Huang ${ }^{3}$, and Yukun Bao ${ }^{1}$ \\ ${ }^{1}$ School of Management, Huazhong University of Science and Technology, \\ Wuhan, 430074, China \\ \{wanglin, jlzhang, yukunbao\} @hust.edu.cn \\ ${ }^{2}$ School of Computer Science and Technology, Hubei University of Economics, \\ Wuhan, 430205, China \\ zengyurong@sohu.com \\ ${ }^{3}$ School of Management, Huazhong University of Science and Technology, \\ Wuhan, 430074, China \\ whuang@ams. .ac.cn
}

\begin{abstract}
This paper presents artificial neural networks (ANNs) for the criticality evaluating of spare parts in a power plant. Two learning methods were utilized in the ANNs, namely back propagation and genetic algorithms. The reliability of the models was tested by comparing their classification ability with a hold-out sample and an external data set. The results showed that both ANN models had high predictive accuracy. The results also indicate that there was no significant difference between the two learning methods. The proposed ANNs was successful in decreasing inventories holding costs significantly by modifying the unreasonable target service level setting which is confirmed by the corresponding criticality class in the organization.
\end{abstract}

\section{Introduction}

Inventory control of spare parts (SPs) plays an increasingly important role in modern operations management. The trade-off is clear: on one hand a large number of spare parts ties up a large amount capital, while on the other hand too little inventory inevitably results in poor supply service level or extremely costly emergency actions [1]. There are about 5,000 SPs that are vital for safely production in a nuclear power plant in China. This plant is one of the most successful commercial power plants and the main technologies are gained from France. In addition, most of them are nonstandard and purchased from France with a lead-time ranging from 8 to 20 weeks. The company is usually obliged to carry inventories consisting of over 110 millions dollars of SPs ready for maintenance.

A key distinguishing feature of the SPs inventory system is the need to specify the criticality of the items. The criticality of an item is a very important factor associated with the service level that can be defined as the probability of no shortage per replenishment from shelf. Then, we can identify the optimal control parameters according to all kinds of constrain condition. Factors such as costs of SPs, availability, storage considerations, probability of requirement of a SP, machine 
downtime costs, etc., are generally considered while managing SPs inventories. Many analytical models of different inventory control systems have been discussed [23].However, there is no evidence that any of the works have attempted to raise the question of evaluating the criticality of SPs using systematic and well-structured procedures. Moreover, the various models described in the literature feature many assumptions that remain violated in real life. Simple and straightforward procedure such as ABC classification approach and the analytic hierarchy process (AHP) analysis have been used in practice for specifying inventory control polices and for fixing inventory review periods [4]. But the index considered, named lead time, type of spare and stock out implication, is so simple and may results in inaccuracy result. A better way to manage an inventory is thorough the development of better technique for identifying the criticality class $(\mathrm{H}, \mathrm{M}$ or $\mathrm{L})$ of a SP, which can also be regard as a classification problem, and management inventories from the point of view of their necessity in maintenance operation. However, the criticality of SPs needs to be evaluated and this is a difficult task which is often accomplished using subjective judgments. Therefore, identifying criticality class for a new SP in an accurate and efficient way becomes a challenge for inventory management.

Artificial neural network (ANN) is an artificial intelligence based technique, which is applicable to the classification process. The ANN can simulate a manager's utilization of perceived relationships for both quantitative and qualitative cues that provide important intermediate steps towards reaching the decision maker's final judgment. These networks have at least two potential strengths over the more traditional model-fitting techniques [5]. First, ANNs are capable of detecting and extracting nonlinear relationships and interactions among predictor variables. Second, the inferred patterns and associated estimates of the precision of the ANN do not depend on the various assumptions about the distribution of variables. The purpose of this study is to examine the classification accuracy of ANN as an aid to facilitate the decision making process of identifying the criticality of SPs. More specifically two types of learning methods, namely back propagation (BP) and genetic algorithms (GA) are used to examine the ANNs classification ability. The rest of this paper is organized as follows. Section 2 reviews the concepts of ANN. This is followed by the research methodology, and the evaluation of the classifier models. The paper concludes with summary of the findings and directions for future research.

\section{Artificial Neural Networks}

Like their biological counterpart, ANNs are designed to emulate the human pattern recognition function by the parallel processing of multiple inputs, and can capture the causal relationships between the independent and dependent variables in any given data set, i.e. ANNs have the ability to scan data for patterns which can be used to construct nonlinear models [6].

An ANN consists of a number of neurons, which are distributed in a number of hierarchical layers. One of the most widely implemented neural network architecture is the multilayer perceptions (MLP) model. A typical MLP used in this paper is shown in Fig. 1. This network has a three-layer, feed forward, hierarchical structure. The total number of neurons, number of neurons on each layer, as well as the number 
of layers determines the accuracy of the network model. The neurons in the input layer represent the attributes or stimuli in a data set. These inputs $\left(x_{1}, x_{2}, \ldots, x_{\mathrm{n}}\right)$ initiate the activations into the network.

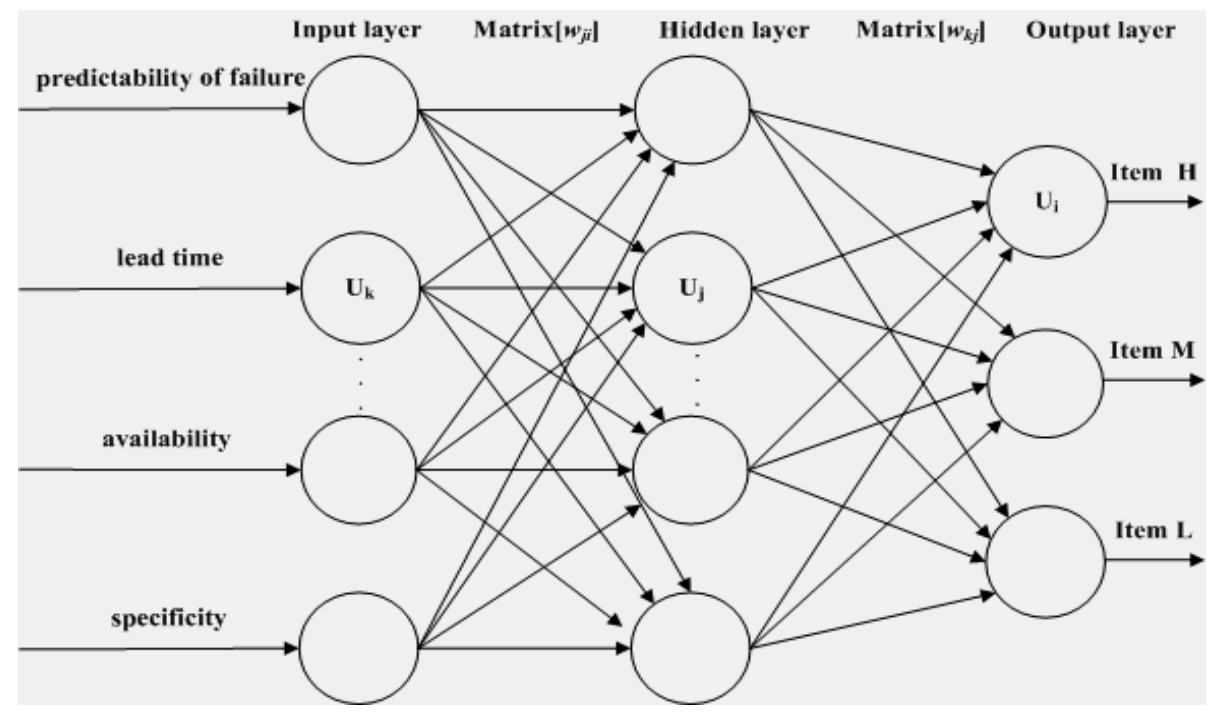

Fig. 1. Structure of the MLP ANN

As illustrated in Fig.1, these inputs are combined in the lower portion of the neuron. The upper portion of the neuron takes this sum and calculates the degree to which the sum is important using a transfer function $(f)$; producing an individual output,

$$
f\left(\sum_{i=1}^{n} w_{i} x_{i}\right)
$$

where, $w$ is weight vector $w=\left[w_{1}, w_{2}, \ldots, w_{n}\right]$; and $x$ is the input vector $x=\left[x_{1}, x_{2}, \ldots, x_{\mathrm{n}}\right]$; for a specific neuron. The transfer function serves as a dimmer switch for turning on and off, depending on the input into the neurons. The selection of the transfer function typically depends on the nature of the output of the network. In this regard, there are a number of alternatives, including the step function, sigmoid function, hyperbolic tangent function, and linear function among others. Because the output of this study is continuous in nature and ranges from 0 to 1 , this study uses the sigmoid transfer function $f\left(w^{\prime} x\right)=1 /\left(1+e\left(-w^{\prime} x\right)\right)$ as recommended by Zahedi [7].

Since the criticality evaluating of SPs problems are inherently non-linear in nature, it is important to create an ANN, which can approximate complex non-linear functions. This is achieved by adding hidden layers (i.e. several layers of sigmoid functions), which consist of neurons that receive inputs from the preceding cells and pass on outputs to subsequent cell layers. Although in theory a single hidden layer is 
sufficient to solve any function approximation problem, some problems may be easier to solve using more than a single hidden layer [8]. A commonly used learning method in $\mathrm{ANN}$ is the BP algorithm.

\subsection{Back Propagation Algorithm Based Learning}

The essence of the BP learning algorithm is to load the input/output relations within the MLP topologies so that it is trained adequately about the past to generalize the future [9]. The steps of the BP algorithm are given below:

Step 1: initialize weights to connections $\left[w_{j k}\right]$ and $\left[v_{i j}\right]$ with random weights.

Step 2: input $X_{i}(i=1 \ldots n)$ receives an input signal and passes the signal to the hidden units $Z_{j}$.

Step 3: each of the hidden units $Z_{j}(i=1, \ldots, n)$ sums the weighted input signals net input to $Z_{j}=\theta_{j}+\sum_{i=1}^{n} x_{i} v_{i j}$ and applies the activation function to compute the output signal, where $\theta_{k}=$ Bias on hidden unit $j$.

Step 4: each of the output units $Y_{k}(k=1, \ldots, n)$ sums the weighted input signals net input to $Y_{k}=\theta_{k}+\sum_{j=1}^{n} z_{j} w_{j k}$ and applies the activation function to compute the output signal, $u_{k}=$ Bias on output unit $k$.

Step 5: each of the output units compares its computed activation with a target to determine association error $e_{k}=y_{k}-t_{k}$ : Based on $e_{k}$, errors at output unit $Y_{k}$ is calculated as follows:

$$
\delta_{k}=e_{k} \theta_{k}+\sum_{j=1}^{n} z_{j} w_{j k}
$$

and sent to all neurons in the previous layers.

Step 6: when the training converges and the system error decreases below an acceptable threshold, the ANN is considered to be trained and then applied over the testing data set.

The BP algorithm however, may not provide the most efficient way to train neural networks and has in many instances resulted in inconsistent performance [10].In other words, obtaining a global solution is often dependent on the choice of starting values. An alternate approach to learning is selections, i.e. a complete behavior system is generated, by evolutionary process. Evolutionary development has been shown to be an extremely important source for generating more complexity in systems. Evolutionary development has been studied in great depth from a mathematical point of view, for instance [11].A common variant for classifying systems is called GA. This learning technique is discussed below.

\subsection{Genetic Algorithm Based Learning}

GA is a stochastic heuristic optimization search technique designed following the natural selection process in biological evolution, i.e. it models the nature of sexual reproduction in which the genes of two parents combine to form those of their 
children. When this technique is applied to problem solving, the basic premise is that an initial population of individuals representing possible solutions to a problem is created. Each of these individuals has certain characteristics that make them more or less fit as members of the population. The most fit members will have a higher probability of mating than lesser fit members, to produce progeny that have a significant chance of retaining the desirable attributes of their parents [12]. This method is very effective at finding optimal or near optimal solutions to a wide variety of problems, because it does not impose many of the limitations required by traditional methods [13-14]. It is an elegant generate-and-test strategy that can identify and exploit regularities in the environment, and converges on solutions that are globally optimal or nearly so. The GA consists of four steps, namely: initialization, reproduction, selection and convergence.

\subsection{Easy-Use Subsystem}

From what discussed above, we can see that this is a rather complex process. However, easy operating is an important measurement rule of any software system. So, we develop this easy-use subsystem to identify the criticality class of SPs. The main user interface of this subsystem, which is built using the Matlab software produced by Mathworks Laboratory Corporation, is shown in Fig. 2.

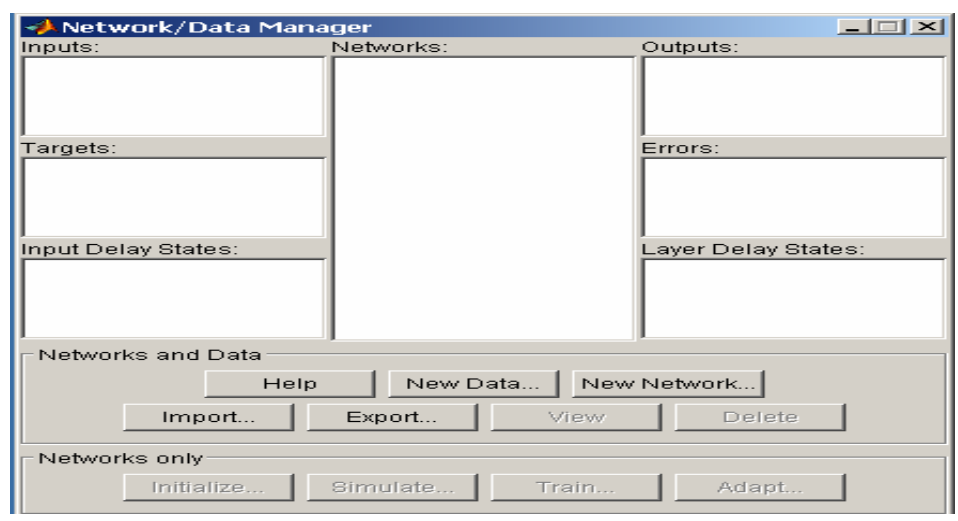

Fig. 2. The user interface of subsystem

\section{Research Methodology}

The empirical investigation was carried out using real-world data obtained from a nuclear power plant in China. A sample of 160 data sets (omitted) was used to design the network. Each data set represented a spare part and contained four types of information: predictability of failure (easy, difficult or impossible), lead time (days, ranging from 8-25 weeks), availability of spare part suppliers (easy or difficult), specificity of a spare part (standard, nor-standard). These criteria were selected based on their importance, as indicated by power plant managers through personal interviews. A five-step procedure was used to design the ANN, as discussed below. 
(1) Representing spare parts in the ANN model

This study represented the identifying the criticality classes of spare parts with regard to four attributes: lead-time, predictability of failure, availability of spare part suppliers and specificity. The first criterion for judging a spare part is lead-time, which is the elapsed time between placing an order with a vendor and when that order arrives. Predictability of failure and specificity of a part is also important for criticality class. At the same time, we know that the criticality class may not be treated as " $\mathrm{H}$ " when the suppliers are easy to find. Inventory managers use ad hoc techniques to integrate the above criteria for criticality class classifications.

(2) Developing the neural network identifying models

Each of the networks consisted of 4 input neurons (one for each spare part characteristic), 16 hidden neurons, and 3 output neurons (namely, criticality class: $\mathrm{H}$, $\mathrm{M}$ or L). The momentum learning parameter was chosen for its simplicity and efficiency with respect to the standard gradient. The value of the learning parameters and the threshold function were kept constant, since the purpose of this study was to evaluate the performance of the ANN topologies.

(3) Training the neural network identifying models

To assess the predictive accuracy of the ANN models the experimental sample was split into two distinct groups, namely, a training group (100 items) and a holdout data (60 items). Using the former, the network models were trained. This process is used to determine the best set of weights for the network, which allow the ANN to classify the input vectors with a satisfactory level of accuracy.

(4) Validating the ANN identifying models

After the network was trained, the holdout data (consisting of the 60 data sets) was entered into the system, and the trained ANN was used to test the selection accuracy of the network for the 60 testing data sets. This is where the predictive accuracy of the machine learning techniques compared against the criticality classes as defined by the experienced inventory managers is measured.

(5) Further validation of the classification models

To validation model's ability to classify data, another data set from another power plant was obtained; this "out of population" i.e. external data set consisting 100 spare parts was input into the predictive model only for purpose of model validation.

\section{Result Analysis and Its Applications}

The results of their classification for 160 randomly selected items are collected. All variables were significantly different for all the groups at $\alpha=0.01$ level, indicating that the three groups represented different populations.

In order to study the effectiveness of the ANN based classifiers, we must test the prediction accuracy by reference to expertise's judgment. As shown in Table 1, some classification errors may be inevitably and have a negative impact on replenishment decision making. So, it is necessary for managers check the classification result before using them. In practice, we can specify different target service level according to the kinds of criticality class. For example, the desire target service level that is measured by the expected shortage per replenishment cycle is specified as $99.95 \%$ for a SP with the criticality class of "H". In the same way, a certain target service level of $97 \%(95 \%)$ is conformed for criticality class of " $M$ ' ("L") according to the practical maintenance requirement. 
Table 1. Prediction accuracy of ANNs (significant at 0.01)

\begin{tabular}{lll}
\hline & ANN $(\mathrm{BP})(\%)$ & ANN (GA) (\%) \\
\hline Overall training sample & 84.5 & 85.8 \\
Holding sample & & \\
Overall classification & 82.4 & 86.9 \\
Item H & 81.9 & 82.1 \\
Item M & 82.6 & 84.2 \\
Item L & 85.2 & 89.6 \\
& & \\
External sample & & \\
Overall classification & 86.0 & 86.3 \\
Item H & 100 & 100 \\
Item M & 84.2 & 85.2 \\
Item L & 82.8 & 83.9 \\
\hline
\end{tabular}

\section{Conclusions}

This paper presents an ANN approach for criticality class evaluation of various SPs. Specifically, two learning methods were utilized in the ANNs, namely, BP and GA. The reliability of the models was tested by comparing their classification ability with a holdout data and an external data set. The results indicate that the ANN classifier models have a relative high predictive accuracy and acceptable. In addition, the results also indicate that there was no significant difference between the two learning methods. The use of the ANN model can prove to be a persuasive analytical tool in deciding whether the criticality class of an SP should be classified as a category $\mathrm{H}, \mathrm{M}$, or L item. By deploying this neural network based model, the unreasonable target service level setting which is confirmed by the corresponding criticality class are modified and the inventory at DaYa Bay Nuclear Power Plant in China consisting of over 100 billion dollars worth of SPs can be reduced by $6.86 \%$ while maintaining the reasonable target service level. However, although these classification models have several advantages, they also have their limitations. For example, the numbers of variables that can be input into these models are limited and many new important qualitative variables may be difficult to incorporate into the models. So, the model should not entirely replace professional judgment.

\section{Acknowledgement}

This research is partially supported by National Natural Science Foundation of China (No.70571025 and No.70401015), Chinese Postdoctoral Science Foundation (No.2005037680) and the Key Research Institute of Humanities and Social Sciences in Hubei Province-Research Center of Modern Information Management. The authors are grateful for the constructive comments of the referees. 


\section{References}

1. Aronis, K.P., Magou, I., Dekker, R., Tagaras, G.: Inventory control of spare parts using a Bayesian approach: a case study. European Journal of Operations Research, 154(2004)730-739

2. Chang, P.L., Chou, Y.C., Huang, M.G.: A $(r, r, Q)$ inventory model for spare parts involving equipment criticality. International Journal of Production Economics, 97(2005) 66-74

3. Kennedy, W.J., Patterson, J.W.: An overview of recent literature on spare parts inventories. International Journal of Production Economics, 75(2002) 201-215

4. Gajpal, P.P., Ganesh, L.S., Rajendran, C.: Criticality analysis of spare parts using the analytic hierarchy process. International Journal of Production Economics, 35(1994)293-297

5. Bishop, C.M.: Neural networks for pattern recognition. New York: Oxford University Press (1995)

6. Cai, K., Xia, J.T., Li, L.T, Gui, Z.L.: Analysis of the electrical properties of PZT by a BP artificial neural network. Computational Materials Science, 34 (2005) 166-172

7. Zahedi, F.: Intelligent systems for business: expert systems and neural networks, California: Wadsworth Publishing (1994)

8. Partovi, F.Y., Anandarajan, M.: Classifying inventory using an artificial neural network approach. Computer \& Industrial Engineering, 40(2002) 389-404

9. Bansal, K., Vadhavkar, S., Gupta, A.: Neural networks based forecasting techniques for inventory control applications. Data Mining and Knowledge Discovery, 2(1998) 97-102

10. Lenard, M., Alam, P., Madey, G.: The applications of neural networks and a qualitative response model to the auditors going concern uncertainty decision. Decision Sciences, 26 (2) (1995) 209-227

11. Varetto, F.: Genetic algorithms applications in the analysis of insolvency risk. Journal of Banking and Finance, 22 (10-11) (1998) 1421-1429

12. Ferentinos, K.P.: Biological engineering applications of feed forward neural networks designed and parameterized by genetic algorithms. Neural Networks, 18(7) (2005)934-950

13. Sexton R.S., Dorsey, R.E., Johnson, J.D.: Toward global optimization of neural networks: a comparison of the genetic algorithm and back propagation. Decision Support Systems, 22 (2) (1998) 171-185

14. Kuo, R.J., Chen J.A.: A decision support system for order selection in electronic commerce based on fuzzy neural network supported by real-coded genetic algorithm. Expert Systems with Applications, 26(2) (2004)141-154 